Military Technical College Kobry El-Kobbah, Cairo, Egypt

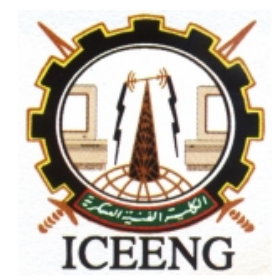

\section{$7^{\text {th }}$ International Conference on Electrical Engineering ICEENG 2010}

\title{
A Strategy to Improve Voltage Instability in The 30-Bus IEEE Power System Model using Remedial Actions
}

$$
\text { By }
$$

S. El-Debieky*

\author{
S. A. Mahmoud***
}

A. H. Hamza**
H. M. Hassanin****

\section{Abstract:}

The analysis of a large interconnection power system, under the severe disturbances such as those that can lead to a voltage collapse (VC), is necessary to develop the performance of the available protection schemes and to avoid critical conditions that can lead to instability or system blackout. In this paper, a Strategy to improve voltage instability in the 30-Bus IEEE power system model as an example to large interconnected power systems. One case study of a large unit tripping is tested and voltage instability was studied. Also, the required remedial actions are considered.

\section{Keywords:}

Voltage Instability, Voltage Collapse (VC), Remedial Actions

* Ain Shams university

** College of Engineering in Shoubra, Benha University, Cairo, Egypt

*** Egyptian Electricity Holding Company

**** Ministry of Electricity and Energy 


\section{Introduction:}

The analysis of a large interconnection power system, under the severe disturbances such as those that can lead to a VC, is necessary to develop the performance of the available protection schemes and to avoid critical conditions that can lead to instability or system blackout [1].

Developments in power systems require increasing amounts of energy transmission from generating stations that are on a far distance from the main load centers. The consequent higher loading on the transmission network has added to the reactive power demands on the interconnected grids, and in turns complicated the difficulties of voltage control. This factor has contributed to the increasing number of worldwide incidents that have led to system voltage instability followed by extensive power system collapses [2].

VC means the loss of power system voltages due to some disturbances [3]. In fact, it is a dangerous phenomenon; it may frequently lead to blackout of all the electrical power system or a major part of it. Wherever the VC occurs the blackout may occurs causing social, economical loss and political problems.

VC phenomenon has been recorded in many advanced countries worldwide: e.g. USA, UK, Sweden, France, and Japan. In most of the reported incidents, VC has been initiated with slow phase, lasting one to four minutes, followed by a second fast phase which swiftly led to system collapse [4].

This type has been labeled as "classic" system voltage instability. There are two other types of system voltage instability, "transient' and "slow", which are significantly different and can be distinguished by the time taken to collapse. VC has been deemed responsible for several major disturbances [5].

Different previous literatures were depicted models to study the phenomenon of system voltage instability. One of these models is the the 30-Bus IEEE Power System Model [6-7]. In this paper, a Strategy to improve voltage instability in the 30-Bus IEEE power system model.

After assuming some modifications on IEEE model like as the current carrying capacity $\left(\mathrm{I}_{\mathrm{ccc}}\right)$, schedule voltage of generation unit $(\mathrm{s})\left(\mathrm{V}_{\mathrm{sch}}\right)$, tap ratio of the tie transformer(s) and the power factor (P.F.); the power system simulation for engineering (PSS/E) software package; was selected to simulate the above mentioned system.

These modifications are applied in order to simulate the low level voltage problem 
which may lead to $\mathrm{VC}$ in case of abnormal conditions such as: contingency due to one $345 \mathrm{kV}$ circuit tripped, contingency due to one $138 \mathrm{kV}$ circuit tripped, the same case while the other circuit is under maintenance, one big generating unit tripped, ... etc.

\section{Voltage Collapse:}

The VC can define as: The collapse of the power system voltage at a certain value called the critical voltage. This occurs in a highly loaded network working too close to the transmission power limit. Also, the heavier line loading results in an increased need for reactive power management increasing the possibilities of voltage instability and collapse. The period of slowly decreasing voltage followed by an accelerating collapse of voltage.

Under adverse operating conditions, a sudden extra reactive demand may exceed the available reactive reserves. This causes a lowering of voltages resulting into transformer tap adjustments that tend to increase further-but the line voltage drops and finally leads to $\mathrm{VC}$. The low voltage situation may be further aggravated by the load characteristics eventually when VC occurs.

A VC is usually accompanied by a sharp rise in the reactive power generation. The effect of allocation and amount of reactive power support on voltage instability margin is based on sensitivity analysis of the generated reactive power with respect to active and reactive load requirements at various locations of the system.

It is important to note that, buses with the largest sensitivities are the ones where even small increase of the load can bring the system to instability. These buses are called critical buses. Also an excessive voltage decline can occur following to some severe system contingencies. This situation could be aggravated possibly leading to $\mathrm{VC}$ by further tripping of more transmission facilities, VAR sources, or generating units due to over loading. In general, the VC may take place as a result of the following situations: a lack of reactive power reserves or control, heavily loaded systems where large amounts of active and reactive power are needed to be transported over long distances, or the sudden increase of the total system load by a small amount in an already heavily loaded system.

A VC may be due to an excessive reactive pick up. This might occur in induction machine loads which have the characteristics that the real power demand tends to be constant as the voltage drops and below a certain voltage the reactive power increases as the voltage drops. 
$\mathrm{VC}$ can be due to the dynamic behavior of the machine components or angle instability of the network. VC takes place if the unbalance between reactive supply and demand cannot be corrected by a limited voltage decrease. Voltage instability generally appears when the electrical distances between voltage regulating devices and loads increase. When very large power systems are to be analyzed, total generated reactive power may be too sensitive to the changes of reactive power of individual loads. The $\mathrm{VC}$ of a power system is analyzed as a result of the eventual push of system operating point beyond the boundary of voltage security due to random fluctuation in the power system load characteristics.

When a generator in a heavily loaded electric power system can become immediately unstable, a dynamic VC leading to blackout may follow. The collapse of the system would result from the loss of steady state stability when generators fall out of synchronism. The existing and continuing trend toward heavier line loading has resulted in an increased need for reactive power management and raised the possibilities of voltage instability and collapse.

\section{VC Methods of Solution:}

In the past, there has been significant confusion over whether the VC problem is a static in nature and therefore be studied as a parametric load flow problem or it is a dynamic and must be studied as a trajectory of a set of dynamic equations. The majority of the work done on this problem has been focused on the static module based on the load flow feasibility. Dynamic VC studies are comparatively less in number due to the complexity involved.

Thomas [3] has proposed the minimum singular value of the Jacobian of the descriptor load flow equations a security index and derived a static control strategy based on that index. Kwatny [8] has studied the static problem as a static bifurcation characterized by the disappearance of the equilibrium point and showed how bifurcation could describe instability both in voltage and angle.

Jung \& Alvarado [9] have proposed a simple algorithm to directly calculate the critical point without numerical difficulties. The method used power flow equations to determine the singularity of the Jacobean matrix. The critical point is the point at which the flow Jacobian is singular but power flow calculations near critical point leads to divergence and error.

Venikov [10] has proved that sign of the determinant of [Jacopian] can be used to determine the operating point is stable or not. The minimum singular value of the power 
flow Jacobian matrix $[\mathrm{J}]$ has been proposed as static voltage instability index (VC security index). The weakest bus is the one that is the nearest to experiencing VC. The weakest bus is one that has a large ratio of $[\mathrm{dv} / \mathrm{dp}]$, where $\mathrm{dv} / \mathrm{dp}$ is the differential change in voltage to differential change in load. Also $\left[-\partial \mathrm{p}_{\text {total }} / \partial \mathrm{v}\right]$ is a good index for system stability, where dptotal is the differential change in active load for the whole system when it is zero, the weakest bus experiences $\mathrm{VC}$ and when it is high the weakest bus is far from instability.

An approximate method to calculate the critical load bus voltage based on the maximum power transfer limit and reduced bus admittance matrices. This critical value is used as a static voltage instability index. An approach to detect the critical load flow causing the $\mathrm{VC}$ of the electric power system. It is based on the gradient method applied in $[\mathrm{P}-\mathrm{Q} / \mathrm{P}-$ $\mathrm{V}]$ space of load flow calculations. The worst load destruction pattern is known through iterative computation of the gradient. The load flow feasibility [LFF] method is very attractive for large power system operators to predict.

The VC proximity has to take proper actions well before it actually happens. Many proposals have considered voltage instability and voltage controllability that are based on the natural cause/ effect relationships that exist at PQ buses in the power system under normal conditions.

Ajjarapu [11] has used the predictor-corrector technique to solve voltage instability problems. The technique depends on finding continuation of power flow solutions starting at some loading base to the steady state voltage instability limit [critical point] of the system. A salient feature of the so-called continuation power flow is that it remains well conditioned at and around the critical point. As a consequence, divergence due to ill conditioning is not encountered at the critical point, even when single precision computation is used. Intermediate results of the process are used to develop a voltage instability index and identify areas of the system most prone to VC. Research is only now beginning to emerge on the dynamics associated with VC. It is clear that the collapse dynamics cannot be described by the generator dynamics, which are traditionally believed to be responsible for transient instabilities.

Power or increase in reactive consumption due to unexpected contingencies. Previous investigations concerned many analysis of this phenomenon through various techniques and computer programs. However, in this study the MATLAB Program module is used for the 30-Bus IEEE Power System Model simulation and for the analysis of VC phenomenon as well. Moreover, the critical VAR load expected at each bus of the system to avoid VC is also determined. 


\section{System Data Analysis:}

After assuming some modifications, IEEE model has been used taking into consideration the following assumptions: the $\mathrm{V}_{\text {sch }}$ of all generating units is nominal value $\left(\mathrm{V}_{\text {sch }}=1.00 \mathrm{p} . \mathrm{u}\right)$ except generation units at ADAMSGEN (B\#1) power plant, it has $\mathrm{V}_{\text {sch }}=0.98 \mathrm{p}$.u to avoid the over voltage problem at this bus, the tap ratio of all tie transformers is set at nominal ratio $(\mathrm{a}=1)$, average power factor is 0.85 , and all cases were carried out at peak load [6]. The objective of the above mentioned assumptions is to make IEEE model similar to the Egyptian power system in its normal conditions at peak load. The 30-Bus IEEE Power System Model is shown in Figure (1).

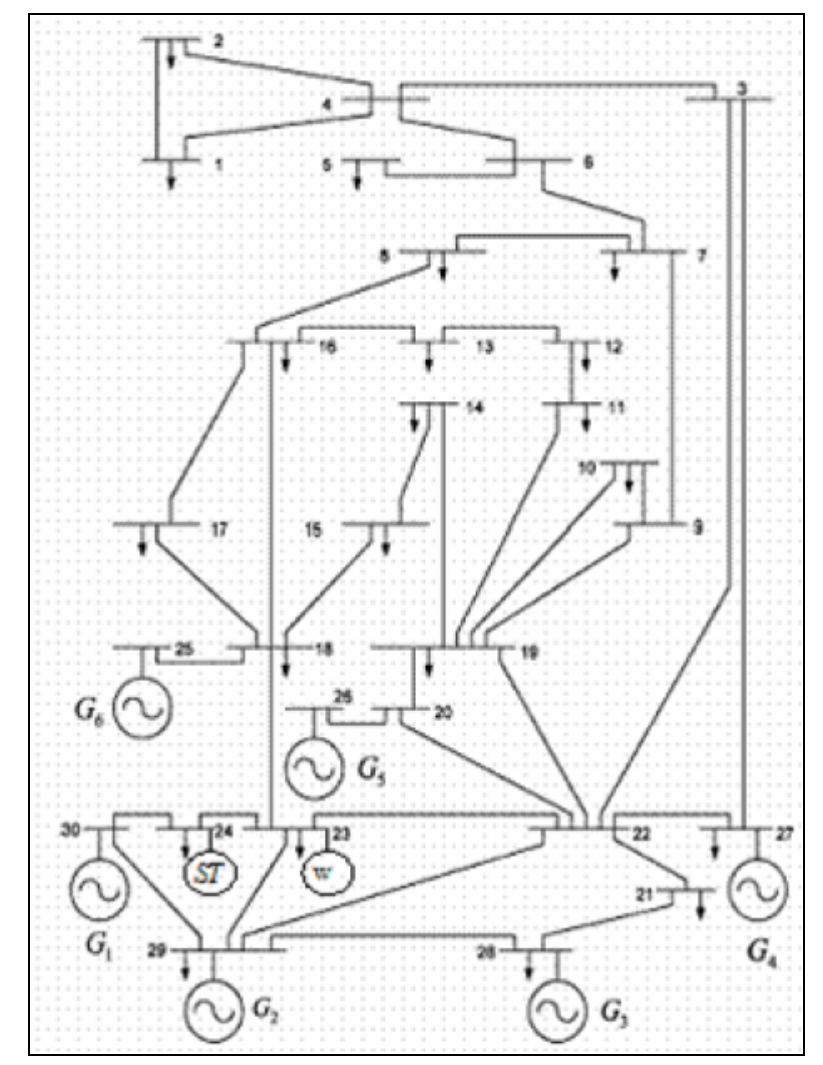

Figure (1): The 30-Bus IEEE Power System Model

Load flow study was carried out for IEEE model network for both normal and emergency conditions in order to identify the voltage profile, transmission lines loading and the transformers lading to know how far the system from the VC. Figure (2) shows that the lines loading within the permissible limits (up to 90\%), however the system is suffering from low voltage level, as it is without the permissible limits $(0.95<\mathrm{V}<1.05$ p.u $)$ which may lead to VC. The voltage levels were as shown in Figure (3). 
An emergency condition is supposed by tripping one generating unit. In this case the system will loss a generating unit at ERNSTGEN (B\#5) which has maximum capacity of $60 \mathrm{MW}$. Due to losing $60 \mathrm{MW}, 138 \mathrm{kV}$ T.L connected between two buses BAKERGEN (B\#2) / ERNSTGEN (B\#5) will be over loaded by $126 \%$, and it will be tripped by the protection devices (over current) as shown in Figure (4). The $138 \mathrm{kV}$ T.L. BAKERGEN (B\#2) / ERNSTGEN (B\#5) will be tripped, causing over loading for many transmission lines and transformers .this will lead to low voltage level at all substation and the system going to have voltage collapse lead to complete black out. As shown in Figure (5). The red lines represent the overloaded parts and the dashed lines represent the off parts.
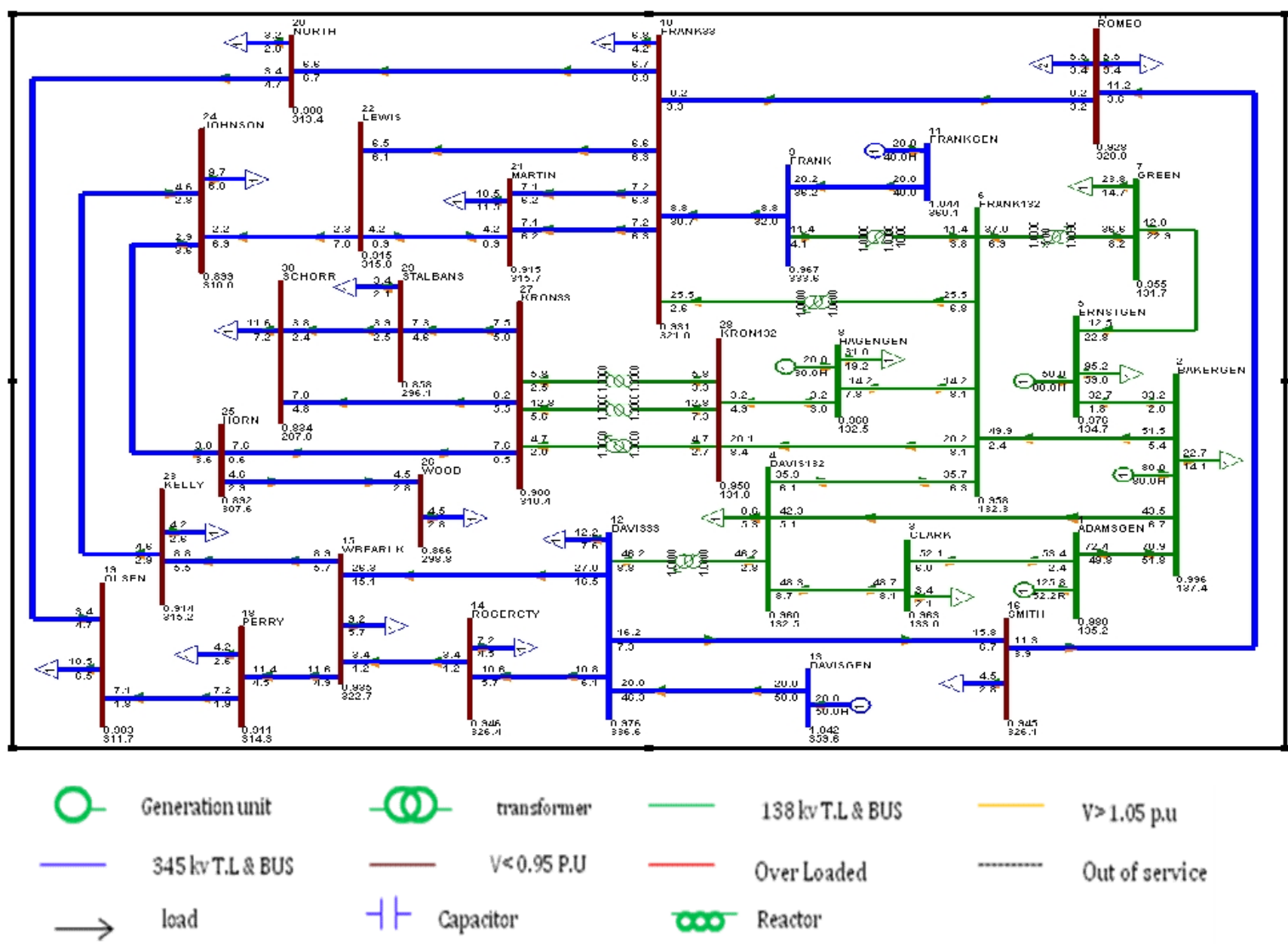

Figure (2): Power Flow for the IEEE Model in Normal Conditions 


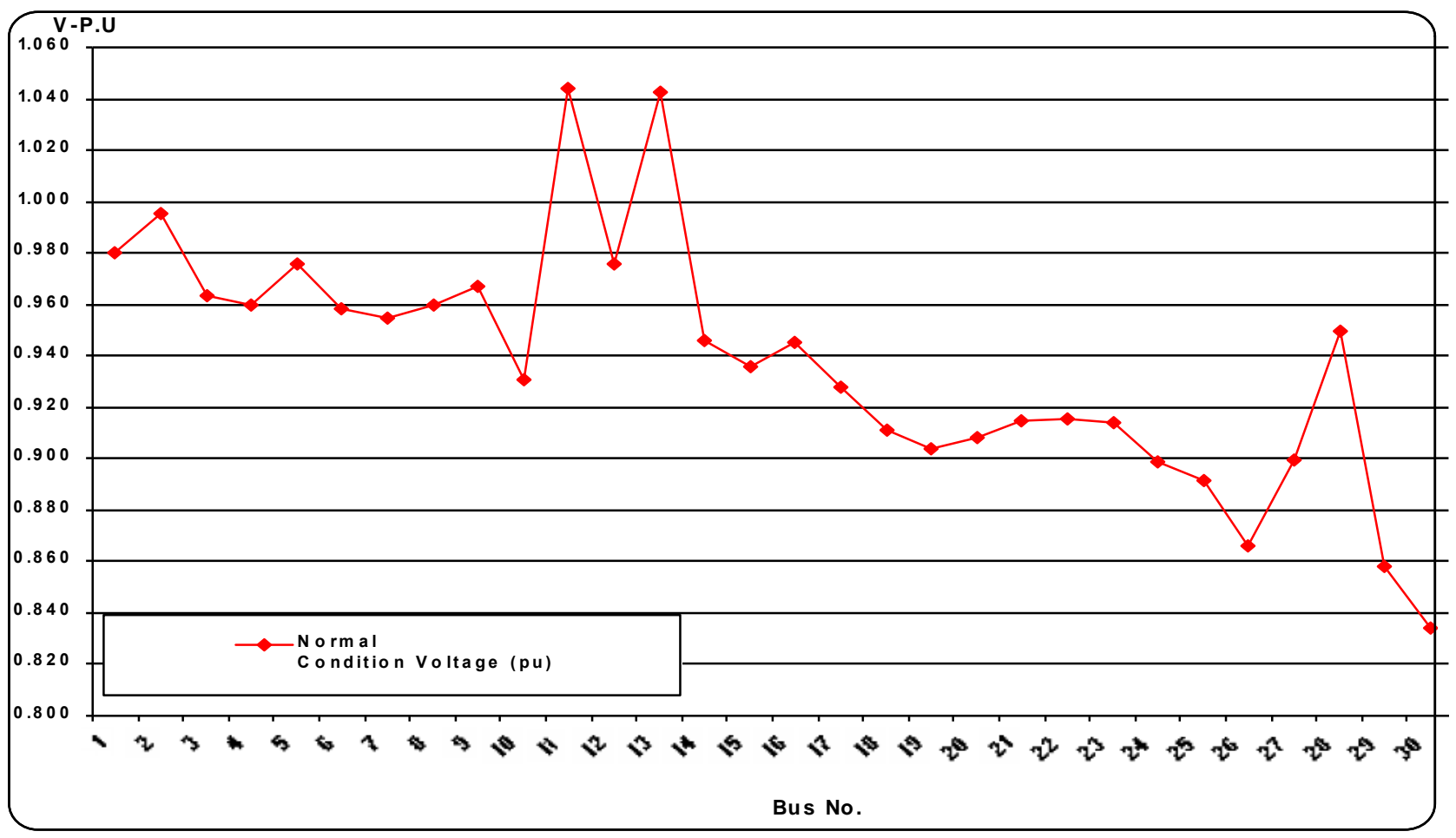

Figure (3): Voltage Levels for IEEE Model in Normal Conditions

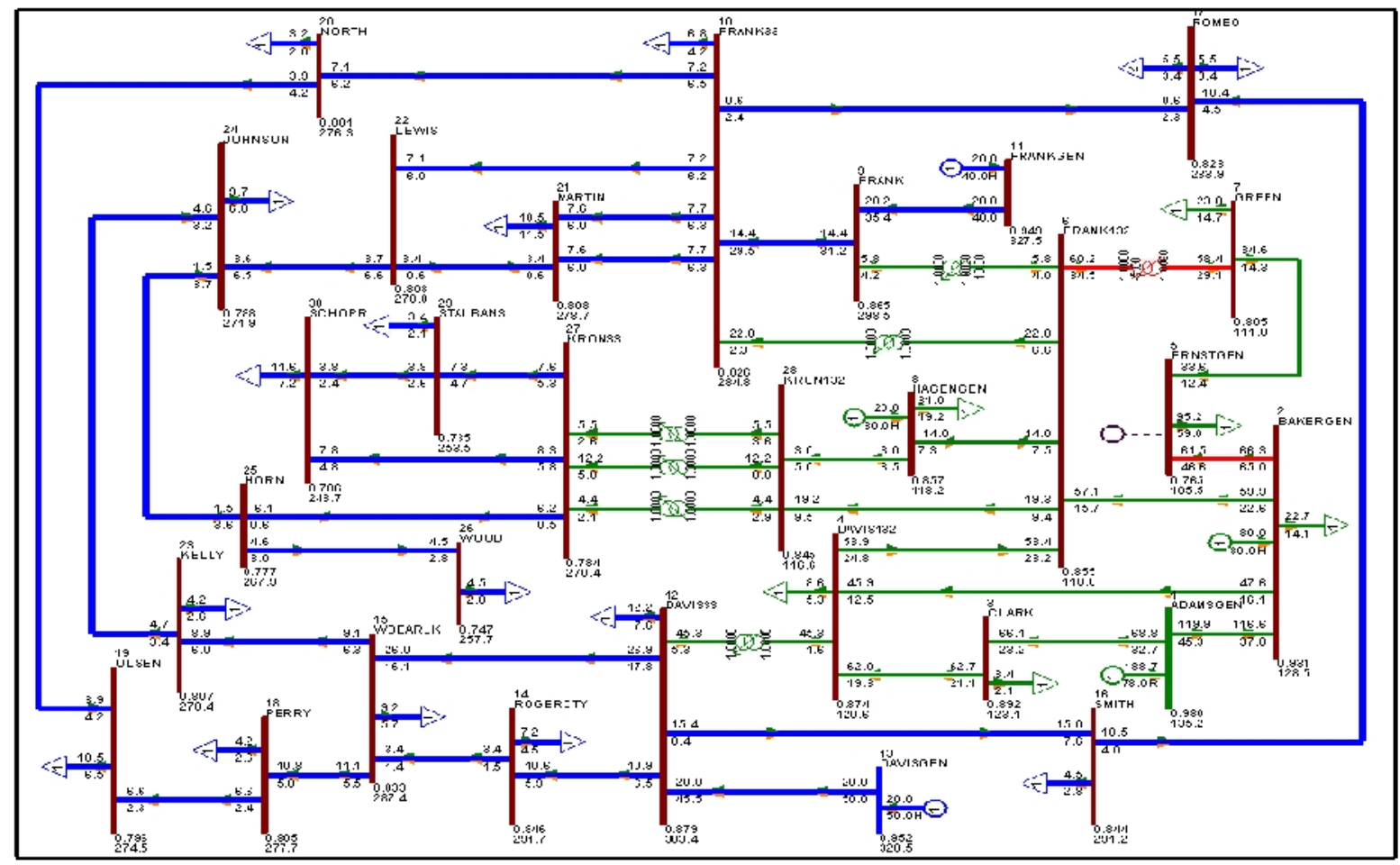

Figure (4): Voltage Level in Case of Different Line Trip Scenarios 


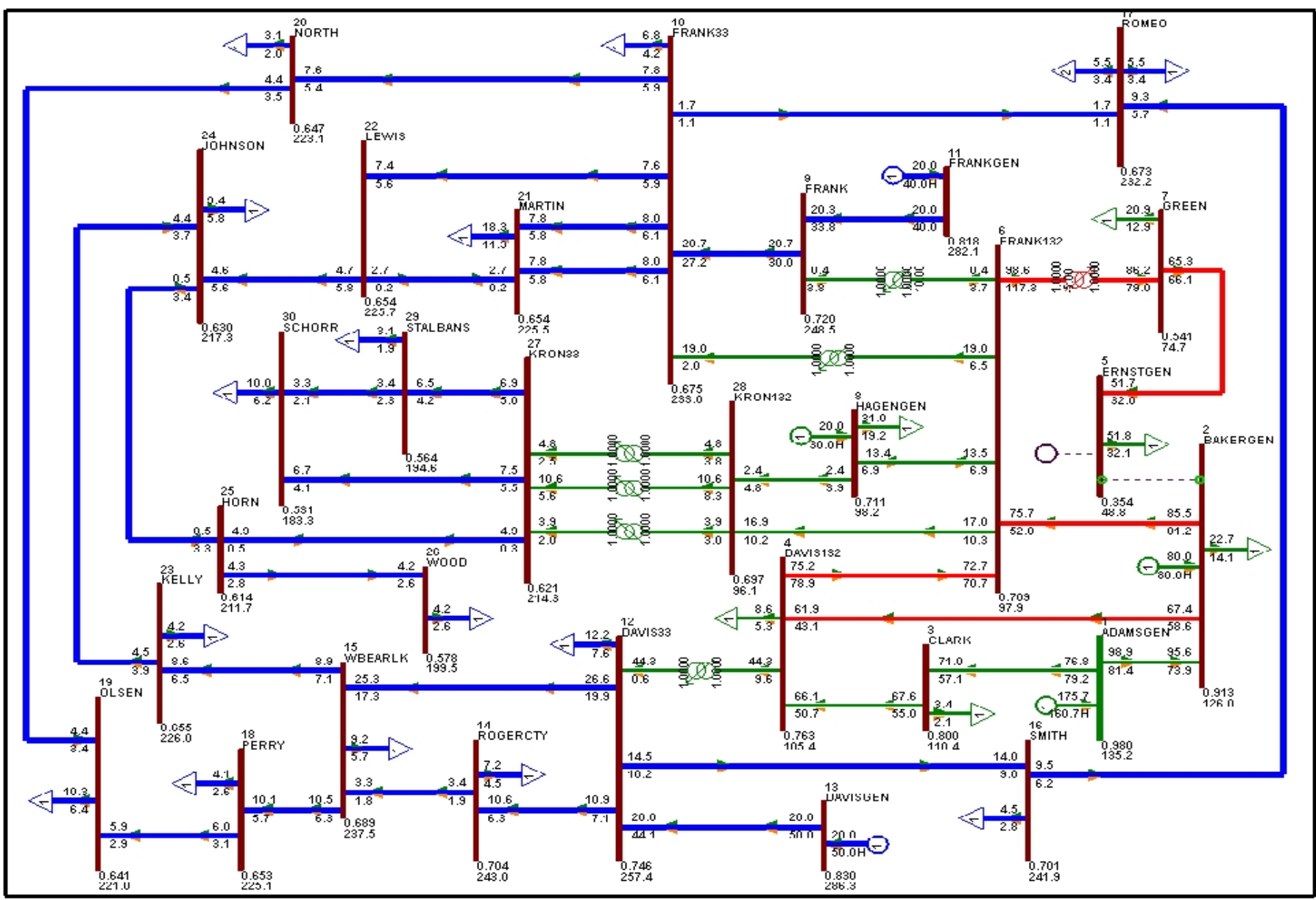

Figure (5): Load Flow in Case of one Generating Unit Trip at (B\#5) and one 138 kV T.L. between Buses (B\#5) and (B\#2)

\section{Remedial Actions:}

Optimal usage of reactive power produced from the generating units; is the remedial action deals with the maximum and minimum real power and reactive power capabilities.

The maximum reactive power capability corresponds to the maximum reactive power that the generator may produce when operating with a lagging power factor. The minimum reactive power capability corresponds to the maximum reactive power the generator may absorb when operating with a leading power factor. 
These limitations are a function of the real power output of the generator that is, as the real power increases, the reactive power limitations move close to zero. The solid curve in Figure (6) is a typical generator capability curve which shows the lagging and leading reactive limitations (the ordinate) real power is varied (abscissa).

Most power flow programs model the generator reactive capabilities by assuming a somewhat conservative value for $\mathrm{P}_{\max }$ (perhaps $95 \%$ of the actual value), and then fixing the reactive limits $\mathrm{Q}_{\max }$ (for the lagging limit) and $\mathrm{Q}_{\min }$ (for the leading limit) according to the dotted lines.

Application of optimal usage of reactive power produced from the generating units using the IEEE model. In the IEEE model, the schedule voltage $\left(\mathrm{V}_{\text {sch }}\right)$ of all generating units is nominal $\left(\mathrm{V}_{\mathrm{sch}}=1.00 \mathrm{p}\right.$.u) except generating unit at ADAMSGEN (B\#1) power plant - it has $\mathrm{V}_{\text {sch }}=0.98 \mathrm{p} . \mathrm{u}$ - to avoid the over voltage problem.

All generating units generates the maximum reactive power except the generating unit at ADAMSGEN (B\#1) which working as a swing bus, so the increase in the schedule voltage which responsible for reactive power generation is useless for other units and the increasing $\left(\mathrm{V}_{\text {sch }}\right)$ the schedule voltage $\left(\mathrm{V}_{\mathrm{sch}}\right)$ at ADAMSGEN (B\#1) Power Plant must be with constrains to avoid the over voltage problem.

Figure (7) illustrates voltage level after increasing the schedule voltage $\left(\mathrm{V}_{\mathrm{sch}}\right)$ of ADAMSGEN (B\#1) power plant from $98 \%$ to $99 \%$ to avoid the over voltage problem.

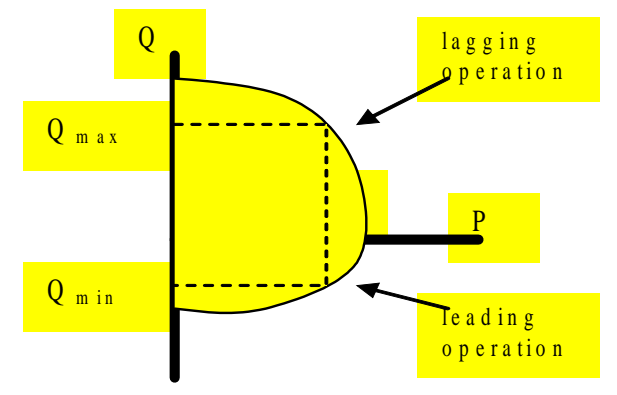

Figure (6): Typical Generator Capability Curve 


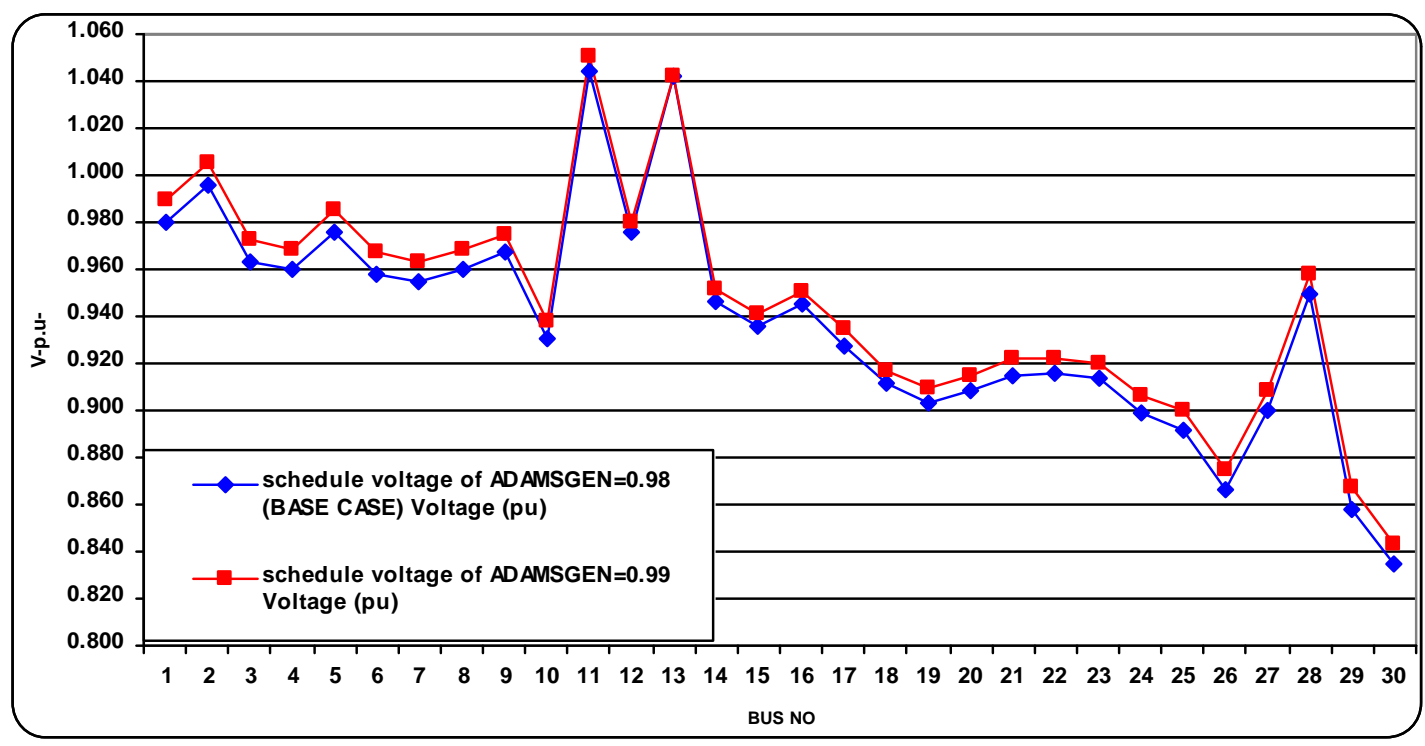

Figure (7): Voltage Levels after Increasing $V_{\text {sch }}$ of Power Plant at (B\#1) from $98 \%$ to $99 \%$

\section{Conclusion:}

In this paper, a Strategy to improve voltage instability in the 30-Bus IEEE power system model as an example to large interconnected power systems. The analysis of such large interconnection power systems, under the severe disturbances such as those that can lead to a voltage collapse (VC), is necessary to develop the performance of the available protection schemes and to avoid critical conditions that can lead to instability or system blackout. One case study of a large unit tripping is tested and voltage instability was studied. The required data and results were illustrated. Also, the required remedial actions to maintain the system voltages within stability margins are considered.

\section{References:}

[1] Voltage Stability of Power Systems: Concepts, Analytical Tools and Industry Experience, IEEE-90TH0358-2-PWR, System Dynamic Performance, Subcommittee of the Power System Engineering, Committee of the Power Engineering society.

[2] M. Z. El Sadek, Preventive Measures for Voltage Collapses and Voltage Failures in the Egyptian Power System, Electric Power System Research, Vol. 44, 1998. 
[3] Thomas J. Overbye, Effective Calculation of Power System Low-Voltage Solutions, IEEE Transaction on Power Systems, Vol. 11, No. 1, Feb. 1996.

[4] Kenji Iba, Hiroshi Suzuki, Masanao Egawa and tsutomuw, A Method for Finding a Pair of Multiple Load Flow Solutions in Bulk Power Systems, IEEE Transaction on Power Systems, Vol. 5, No. 2, May 1990.

[5] P. Kundur, G. J. Rogres, D. Y. Wong, L. Wang and M. G. Lau, A Comprehensive Computer Program Package for Small Signal Stability Analysis of Power Systems, IEEE Transaction on Power Systems, Vol. 5, No. 4, Nov. 1990.

[6] Mohammad Shahidehpour and Yaoyu Wang, Communication and Control in Electric Power Systems, John Wiley \& Sons, Inc., 2005.

[7] A. Arunagiri and B. Venkatesh, Simulation of Voltage Stability and Alleviation Through Knowledge Based System, American Journal of Applied Sciences, Vol. 1, No. 4, 2004.

[8] H. Kwatny, Saddle-Node Bifurcation of Voltage Profiles of Small Integrated AC/DC Power Systems, IEEE 2000, Center for Electric Power Engineering, Drexel University, Philadelphia, PA 19104, USA.

[9] Alvarado, Computation of Closest Bifurcations in Power Systems, IEEE Transaction on Power Systems. Vol. 9, No. 2, May 1994.

[10] V. Venikov, Transient Processes in Electrical Power Systems, Mir Publishers, Moscow.

[11] Ajjarapu, An Optimal Reactive Power Planning Strategy Against Voltage Collapse, IEEE Transaction on Power Systems, Vol. 9, No. 2, May 1994. 